\title{
Bleomycin Binding Sites on Alveolar Macrophages
}

\author{
Elizabeth M. Denholm and Sem H. Phan \\ Division of Hematology, Albany Medical College, Albany, New York (E.M.D.), \\ Department of Pathology, University of Michigan, Ann Arbor (S.H.P.)
}

\begin{abstract}
Previous work has demonstrated that bleomycin can directly stimulate alveolar macrophage secretion of fibroblast growth factors and monocyte chemotactic factors. In this study, rat alveolar macrophages obtained by bronchoalveolar lavage were examined for the presence of bleomycin binding sites, which might mediate this response. The results indicated that alveolar macrophages have specific, saturable, and reversible binding sites. Both high- and low-affinity binding sites were found; each macrophage possessed $6.7 \times 10^{4}$ high-affinity sites, with a $K_{d}$ of $528 \mathrm{nM}$, and $2.2 \times 10^{6}$ low-affinity sites, with a $K_{d}$ of $65 \mu \mathrm{M}$. The $K_{d}$ of the high-affinity sites corresponds closely to the ED $D_{s 0}$ obtained from dose-response curves of the bleomycin-stimulated secretion of both fibroblast growth and monocyte chemotactic factors, suggesting that bleomycin stimulation of alveolar macrophage function responses may be mediated by bleomycin interaction with these sites.
\end{abstract}

Key words: pulmonary fibrosis, lung, fibroblast growth factors, monocyte chemotactic factors

\section{INTRODUCTION}

Pulmonary fibrosis induced by the administration of bleomycin in a number of rodent species has been established as an animal model of the human disease [11]. Alveolar macrophages isolated from animals with bleomycin-induced fibrosis secrete cytokines $[6,15]$, fibroblast growth $[2,4,8,11]$ and inhibitory factors [3], and leukocyte chemotactic factors [5,7], all of which are thought to play important roles in mediating the fibrotic reaction of the lungs to this drug. It has also recently been demonstrated that similar results can be obtained by the in vitro stimulation with bleomycin of alveolar macrophages from normal animals $[4-6,15]$.

The experiments presented here were undertaken to determine if alveolar macrophages possess specific binding sites for bleomycin. Bleomycin internalization by both tumor and normal cells has been established directly by studies of drug uptake $[9,13]$ and indirectly by the demonstration of chromosomal damage following the incubation of cells with this drug $[1,12,17]$. However, evidence that this internalization is a receptor-mediated event has been lacking. In support of this hypothesis, it has been demonstrated that the lethal effects of bleomycin on Chinese hamster ovary (CHO) cells can be ablated by pretreatment of cells with trypsin, implying the presence of an intermediary membrane protein(s) involved in the interaction of this drug and $\mathrm{CHO}$ cells [1]. As described here, the finding of bleomycin binding sites on alveolar macrophages not only suggests a mechanism by which these cells may be stimulated to secrete monokines in bleomycin-induced pulmonary fibrosis but may also indicate a mode of action of this drug that is in addition to its well known cytotoxic effects, mediated by DNA strand breakage $[1,11,12,17]$.

\section{MATERIALS AND METHODS \\ Materials}

Bleomycin (Blenoxane) was a gift from Bristol Laboratories (Bristol-Myers Company, Evansville, IN). [S-methyl- ${ }^{3} \mathrm{H}$ ]-bleomycin $\mathrm{A}_{2}$, copper form $(63-74 \mathrm{Ci} /$ mmol), was purchased from New England Nuclear (Boston, MA). High-pressure liguid chromatography (HPLC)-grade $\mathrm{CH}_{3} \mathrm{CN}$ and $\mathrm{CF}_{3} \mathrm{COH}$ were obtained from Pierce (Rockford, IL). Trypan blue and Wright's stain solution were from Sigma Chemical Co. (St. Louis, MO). All other chemicals used to prepare buffers were of reagent grade or better and were purchased from Fisher Scientific Co. (Livonia, MI).

\section{Methods}

Purification of bleomycin $\mathbf{A}_{2}$. Blenoxane is a mixture of bleomycin $A_{2}$ and $B_{2}$ [15]; for use in binding assays, bleomycin $A_{2}$ was purified using reverse-phase HPLC with a Waters Associates 510 chromatograph system (Waters, Div. of Millipore, Milford, MA). The column was an RP 318 (Biorad, Richmond, CA), with 3

Received January 8, 1990; accepted April 7, 1990.

Reprint requests: Elizabeth $M$. Denholm, Division of Hematology (A52), Albany Medical College, 47 New Scotland Avenue, Albany, NY 12208. 
$\mu \mathrm{m}$ particle size and measuring $4.6 \times 250 \mathrm{~mm}$. Elution was accomplished isocratically at $22^{\circ} \mathrm{C}$ with $89 \%$ solvent A $\left(0.1 \% \mathrm{CF}_{3} \mathrm{CO}_{2} \mathrm{H}\right.$ in $\left.\mathrm{H}_{2} \mathrm{O}\right)$ was $11 \%$ solvent $\mathrm{B}(0.1 \%$ $\mathrm{CF}_{3} \mathrm{CO}_{2} \mathrm{H}$ in $\mathrm{CH}_{3} \mathrm{CN}$ ) and a flow rate of $1.0 \mathrm{ml} / \mathrm{min}$. Under these conditions, as detected by absorbance at 214 $\mathrm{nm}$, bleomycin $\mathrm{A}_{2}$ and $\mathrm{B}_{2}$ eluted at 20 and $31 \mathrm{~min}$, respectively. Material eluting at $20 \mathrm{~min}$ from ten column runs was collected using a fraction collector and pooled, dried using vacuum centrifugation (Speed Vac, Savant Instruments Inc., Hicksville, NY), and resuspended in $95 \%$ ethanol, and stored at $-20^{\circ} \mathrm{C}$. Using this chromatography method, the purity of each commercially purchased batch of $\left[{ }^{3} \mathrm{H}\right]$ bleomycin $\mathrm{A}_{2}$ was also checked before use and was found to consist of $\geqslant 97 \%$ bleomy$\operatorname{cin} \mathrm{A}_{2}$.

Alveolar macrophage isolation. Macrophages were obtained from male-specific pathogen-free Fisher 344 rats weighing 150-200 g (Charles River, Portage, MI) exactly as reported previously [4]. Briefly, rats were killed with an overdose of ketamine, and lungs were perfused with $50 \mathrm{ml}$ of sterile phosphate-buffered saline (PBS) containing $1 \mathrm{mM}$ EDTA and $0.1 \%$ glucose to remove blood. Lungs were then lavaged with ten, $10 \mathrm{ml}$ washes of this sterile PBS solution. Following isolation, macrophages were resuspended in Hank's balanced salt solution (HBSS) supplemented with $1 \mathrm{mM} \mathrm{CaCl}, 1 \mathrm{mM}$

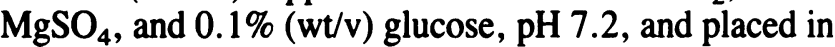
an ice water bath. These cells were $\geqslant 98 \%$ macrophages by analysis of cytocentrifuge (Shandon Instruments, Pittsburgh, PA) preparations stained with Wright's stain. Viability in all cases was $>95 \%$ as assessed by exclusion of trypan blue.

\section{Bleomycin Binding Assays}

Both radioactive and unlabeled bleomycin $\mathrm{A}_{2}$ were dried using vacuum centrifugation to remove ethanol in stock solutions, then resuspended in supplemented HBSS. Polypropylene microfuge tubes (Sarstedt, Princeton, NJ) were siliconized with Prosil-28 (PCR Inc., Gainesville, FL) $24 \mathrm{hr}$ prior to use. Cells, buffers, and bleomycin were chilled to $4^{\circ} \mathrm{C}$ before use, and all incubations with ligand were done at $4^{\circ} \mathrm{C}$. Each tube contained $1 \times 10^{6}$ macrophages and the indicated concentration of $\left[{ }^{3} \mathrm{H}\right]$ bleomycin. Nonspecific binding was determined as dpm bound in the presence of a 100-fold concentration of unlabeled bleomycin $\mathrm{A}_{2}$. For kinetic studies, incubations were at $4^{\circ} \mathrm{C}$ for the times indicated; for all other experiments, the incubations were for 60 min. All incubations were stopped by rapid filtration and washing of cells with ice-cold HBSS using a Brandel cell harvester (Gaithersburg, MD). To reduce nonspecific binding of the radiolabeled ligand, GF/B filters used in harvesting cells were presoaked in $1 \%(\mathrm{wt} / \mathrm{v})$ powdered milk (Carnation) in HBSS just prior to use. All tubes were washed eight times with $1.5 \mathrm{ml}$ of ice-cold HBSS.
Filters were dried and placed into scintillation vials and counted for radioactivity after the addition of scintillant (Ready Protein; Beckmann Instruments, Fullerton, CA).

Analysis of radioactivity bound to macrophages. Four million to six million macrophages per tube were incubated with $169 \mathrm{nM}(0.25 \mu \mathrm{g} / \mathrm{ml})$ of $\left[{ }^{3} \mathrm{H}\right]$ bleomycin for 1 hour at $4^{\circ} \mathrm{C}$ as described above. Following the incubation, tubes were centrifuged $(1,500 \mathrm{rpm})$ for $5 \mathrm{~min}$ at $4^{\circ} \mathrm{C}$, and the supernatant was discarded. Cell pellets were then resuspended in $1 \mathrm{ml}$ ice-cold HBSS and washed thrice by centrifugation; after the last wash, $50 \mu \mathrm{l}$ of $6.77 \mathrm{mM}(10 \mathrm{mg} / \mathrm{ml})$ nonradioactive bleomycin $\mathrm{A}_{2}$ was added followed by $500 \mu \mathrm{l}$ of $5 \%$ trichloroacetic acid (TCA) $\left(4^{\circ} \mathrm{C}\right)$ and vortexed. After $15 \mathrm{~min}$ on ice, TCAprecipitable protein was pelleted by centrifugation. The resultant supernatants were filtered $(0.2 \mu \mathrm{m}$ Acro LC13 HPLC filters; Gelman Sciences, Ann Arbor, MI) prior to analysis, using the HPLC method described above. Peak absorbance of unlabeled bleomycin $A_{2}$ added to the samples was compared to the elution time of radioactivity in the fractions. To ensure that $\left[{ }^{3} \mathrm{H}\right]$ bleomycin was precipitated and unaltered by TCA, $\left[{ }^{3} \mathrm{H}\right]$ bleomycin was incubated in the absence of macrophages then treated with TCA as described for cell samples. Binding data were analyzed using the LIGAND computer program [10] to determine the number and affinity of the binding sites.

\section{RESULTS}

When alveolar macrophages were incubated with concentrations of $20-474 \mathrm{nM}(0.3-0.7 \mu \mathrm{g} / \mathrm{ml})$ of $\left[{ }^{3} \mathrm{H}\right]-$ bleomycin, total binding increased with the concentration of bleomycin present, saturating between 200 and 474 nM (Fig. 1). Nonspecific binding, in the presence of a 100 -fold excess unlabeled bleomycin, increased linearly with increasing concentrations of radioactive ligand. The specific binding, represented as the total minus the nonspecific radioactivity bound, increased in linear fashion from 20 to $169 \mathrm{nM}$ and did not increase further at doses between 169 and $474 \mathrm{nM}$. Alveolar macrophages thus appear to have specific and saturable membrane binding sites for bleomycin $\mathrm{A}_{2}$.

To determine the kinetics of bleomycin binding to these sites, macrophages were incubated with a saturating concentration of $\left[{ }^{3} \mathrm{H}\right]$ bleomycin $(338 \mathrm{nM})$ in the presence or absence of $3.38 \mu \mathrm{M}$ of unlabeled bleomycin for 2-60 min. Binding of the ligand to macrophages was rapid, approaching maximal levels after $10 \mathrm{~min}$ (Fig. 2). Although there was some increase in specifically bound radioactivity from 10 to $80 \mathrm{~min}$, this difference was found not to be statistically different in any of the three experiments performed.

The reversibility of bleomycin binding to macrophages was examined by incubating cells for $60 \mathrm{~min}$ with 


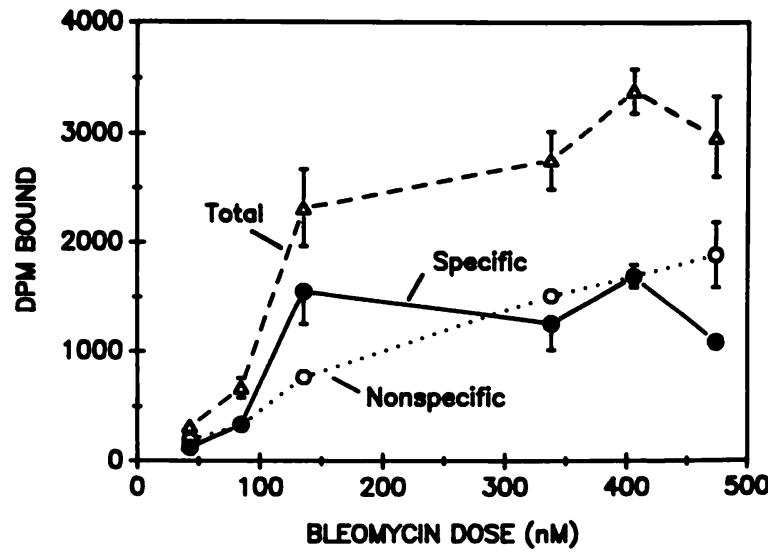

Fig. 1. Dose response of bleomycin binding. Macrophages were incubated at $4^{\circ} \mathrm{C}$ for $60 \mathrm{~min}$ with the indicated concentration of [ ${ }^{3} \mathrm{H}$ ]bleomycin $A_{2}$ in the presence (open circles) or absence (triangles) of a 100-fold excess unlabeled bleomycin $A_{2}$. Specific binding (solid circles) was determined by subtracting nonspecific binding from total binding. Data represent the mean \pm SE (of triplicate data points) of a representative experiment from a total of 10 such experiments.

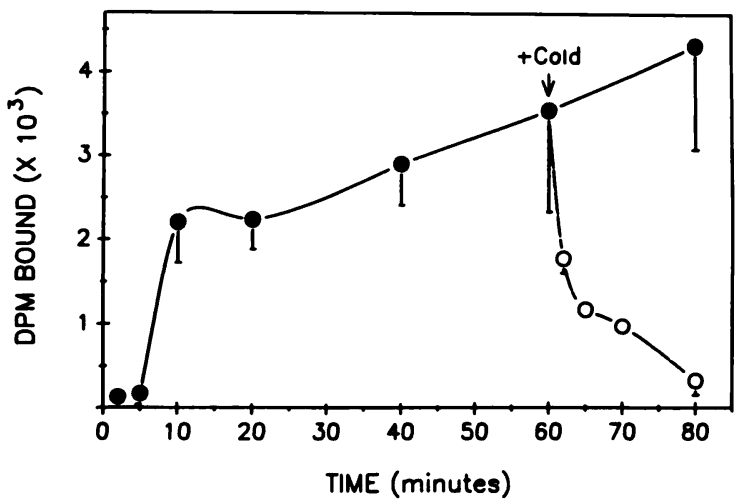

Fig. 2. Kinetics of bleomycin binding. Macrophages were incubated at $4^{\circ} \mathrm{C}$ for the indicated times with $338 \mathrm{nM}$ [ ${ }^{3} \mathrm{H}$ ]bleomycin $\mathrm{A}_{2}$. Specific binding was determined as described in the legend to Figure 1. To examine reversibility, one set of tubes was incubated for 60 min with [ ${ }^{3} \mathrm{H}$ ]bleomycin $A_{2}$ prior to the addition of unlabeled bleomycin (arrow); specific binding remaining at 2, 5, 10, and 20 min after this addition was then determined (broken line). Data represent the mean \pm SE (of triplicate data points) from a total of three such experiments.

$\left[{ }^{3} \mathrm{H}\right]$ bleomycin, then adding a 100 -fold excess unlabeled ligand for an additional time of 2-20 min (Fig.2, broken line). $\left[{ }^{3} \mathrm{H}\right]$ bleomycin was rapidly displaced from binding sites, with a reduction in specifically bound radioactivity evident as early as 2 min after addition of the unlabeled drug; this displacement was essentially complete in 20 min.

The characteristics of these binding sites were analyzed by the ability of increasing doses of cold ligand to displace the binding of radioactive ligand. This was

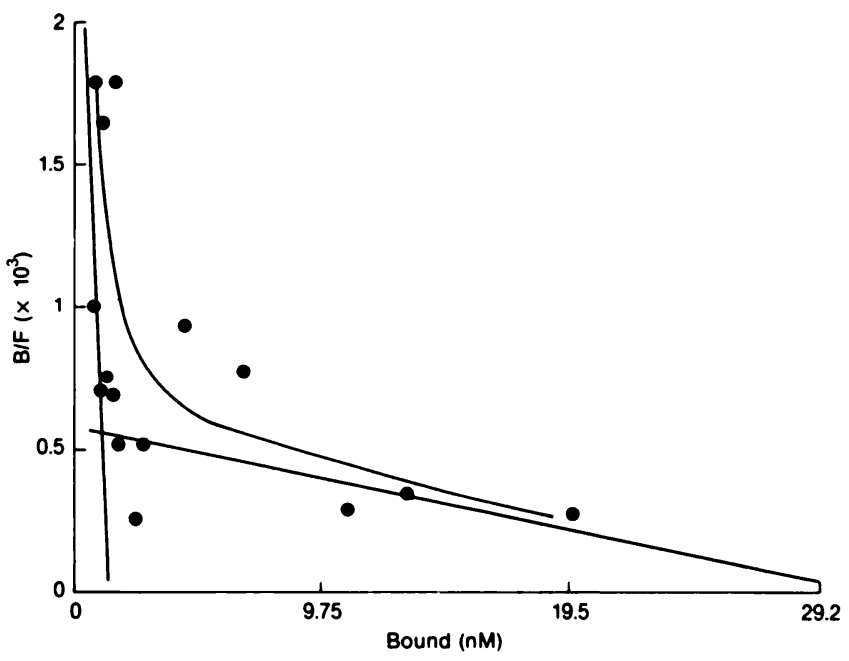

Fig. 3. Scatchard plot of bleomycin binding. Macrophages were incubated at $4^{\circ} \mathrm{C}$ for $60 \mathrm{~min}$ with the indicated total concentration of bleomycin, a constant $271 \mathrm{nM}$ of which was $\left[3 \mathrm{H}\right.$ ]bleomycin $A_{2}$. Bound radioactivity was converted to nmoles; data are the means of duplicate data points from four experiments.

accomplished by incubating alveolar macrophages with a constant concentration of $271 \mathrm{nM}\left[{ }^{3} \mathrm{H}\right]$ bleomycin and increasing concentrations of cold ligand. Analysis of the data from four such experiments by LIGAND revealed the presence of both high- and low-affinity binding sites (Fig. 3). Each macrophage possessed an average of $6.7 \times 10^{4}$ sites, with an apparent $K_{d}$ of $528 \mathrm{nM}$, and an average of $2.2 \times 10^{6}$ low-affinity sites, with an apparent $\mathrm{K}_{\mathrm{d}}$ of $65 \mu \mathrm{M}$. Although low-affinity binding sites were evident in all such experiments, it still remains a possibility that these sites represented nonspecific binding to the plasma membrane.

Finally, HPLC analysis of radioactive bleomycin bound to macrophages under the binding assay conditions described $\left(60 \mathrm{~min}, 4^{\circ} \mathrm{C}\right)$ verified that $\left[{ }^{3} \mathrm{H}\right]$ bleomycin $\mathrm{A}^{2}$ was not metabolized or degraded during the assay period (Fig. 4A). Ninety percent of the radioactivity recovered from macrophages incubated with $\left[{ }^{3} \mathrm{H}\right]$ bleomycin coincided with peak absorbance of nonlabeled bleomycin $\mathrm{A}_{2}$. In parallel controls of $\left[{ }^{3} \mathrm{H}\right]$ bleomycin incubated in the absence of macrophages, $91 \%$ of the radioactivity recovered eluted along with the unlabeled ligand (Fig. 4B).

\section{DISCUSSION}

The results of this study revealed that bleomycin could bind to alveolar macrophages in a rapid, specific, and saturable manner. Since all experiments were performed at $4^{\circ} \mathrm{C}$, it is likely that cell-associated radioactivity reflected bleomycin bound to membrane sites on the cell surface rather than inside the cell. This conclusion is 

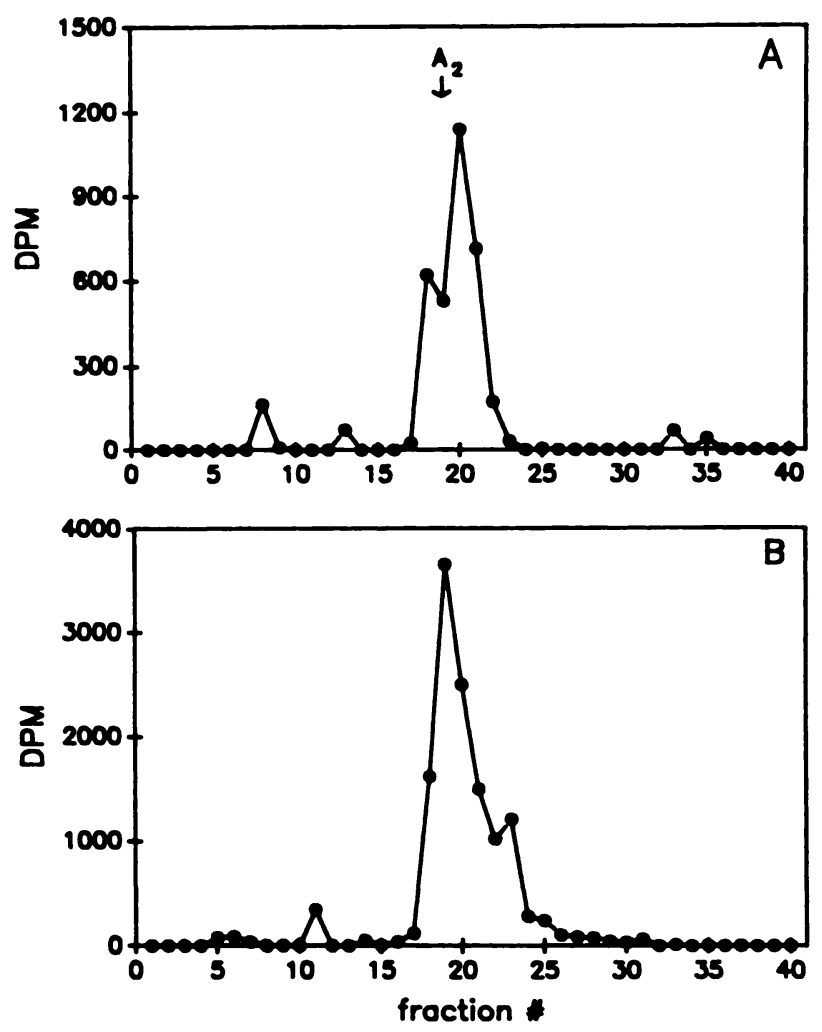

Fig. 4. HPLC analysis of radioactivity bound to macrophages. [3H]bleomycin was incubated with (A) or without (B) macrophages for $60 \mathrm{~min}$ at $4^{\circ} \mathrm{C}$, after which cells were washed to remove unbound ligand. Unlabeled bleomycin was added as a tracer and to enhance dissociation of ligand from cells. [ ${ }^{3} \mathrm{H}$ ]bleomycin recovered in supernatants following TCA precipItation was isolated by HPLC as decribed in Methods. Radioactivity (dpm) and absorbance were determined for each fraction. Arrow indicates absorbance $(214 \mathrm{~nm})$ peak of unlabeled bleomycin $\mathbf{A}_{2}$.

supported by the rapid reversibility of binding (Fig. 2) and by studies with Ehlrich cells [9], which have shown that the association of this drug with cells was irreversible once it was internalized. Furthermore, studies on the effects of temperature on the endocytosis of ligands by alveolar macrophages [16] have indicated that internalization is negligible at $4^{\circ} \mathrm{C}$. The findings presented here are therefore compatible with the existence of bleomycin binding sites on alveolar macrophages.

This demonstration of bleomycin binding sites on macrophages is significant in that the $K_{d}$ of the highaffinity sites $(528 \mathrm{nM})$ is within the concentration range of the doses of bleomycin which evoke a biological response. Previous work in this laboratory has shown that alveolar macrophages obtained from normal rats incubated with $6.77-670 \mathrm{nM}$ bleomycin in vitro secreted both monocyte chemotactic factor and macrophage-derived growth factor (MDGF) [4,5]. Jordana et al. [6] have likewise demonstrated enhanced secretion of interleu- kin-1 by alveolar macrophages incubated with $339 \mathrm{nM}$ bleomycin. Time course experiments have also shown that the kinetics of $\left[{ }^{3} \mathrm{H}\right]$ bleomycin binding to macrophages (Fig. 2) are in accordance with the time course of the secretion of both MDGF and monocyte chemotactic factor, which were detectable as early as $\mathbf{3 0}$ min following stimulation with bleomycin in vitro [4] (also, unpublished observations). The differences in the optimal concentrations of bleomycin necessary for measuring binding and that needed to elicit a biological response may be due to the differences in the incubation temperatures used; binding assays were carried out at $4^{\circ} \mathrm{C}$ whereas experiments on the effects of bleomycin on the secretion of MDGF and chemotactic activity $[4,5]$ were carried out at $37^{\circ} \mathrm{C}$.

Since bleomycin is a glycopeptide product of Streptomyces verticullus [16], it is unclear why alveolar macrophages would have specific binding sites for this drug. Binding sites for such glycopeptides may serve in a protective capacity similar to that suggested for the receptors for bacterial peptides [14] and lipopolysaccharide [18], allowing alveolar macrophages to respond to airborne mold products. It also seems likely that the bleomycin binding sites represent membrane sites with other functions that can also bind to bleomycin; these binding sites may be nonspecific, reacting with a range of glycosalated compounds [16], or specific receptors for some endogenous mediator(s) capable of regulating macrophage function in both homeostasis and disease [11]. The demonstration of these binding sites provides important support for the existence of novel cellular and tissue effects of this drug in addition to its well-known effects on cytotoxicity and DNA strand scission [17].

\section{ACKNOWLEDGMENTS}

This work was supported by National Institutes of Health grants HL28737, HL31963, HL39925, HL07517, and HL42622. Part of this work was performed during the tenure of an established investigatorship (to SHP) from the American Heart Association. The authors thank Frances M. Wolber and Judith A. Snyder for their excellent technical assistance.

\section{REFERENCES}

1. Barranco, S.C., Bolton, W.E. and Noval, J.K. Time-dependent changes in drug sensitivity expressed by mammalian cells after exposure to trypsin. J. Natl. Cancer Inst. 64,913, 1980.

2. Chandler, D.B., Hyde, D.M., and Giri, S.N. Morphometric estimates of infiltrative cellular changes during the development of bleomycin-induced pulmonary fibrosis in hamsters. Am. J. Pathol. 112,170, 1983.

3. Clark, J.G., Kostal, K.M., and Marino, B.A. Bleomycin-induced pulmonary fibrosis in hamsters: An alveolar macrophage product increases fibroblast prostaglandin $\mathrm{E}_{2}$ and cyclic adenosine mono- 
phosphate and suppresses fibroblast proliferation. J. Clin. Invest. 72,2082, 1983.

4. Denholm, E.M., and Phan, S.H. The effects of bleomycin on alveolar macrophage growth factor secretion. Am. J. Pathol. 134,355, 1989.

5. Denholm, E.M., Wolber, F.M., and Phan, S.H. Secretion of monocyte chemotactic activity by alveolar macrophages. Am. J. Pathol. 135,571, 1989.

6. Jordana, M., Richards, C., Irving, L.B., and Gauldie, J. Spontaneous in vitro release of alveolar-macrophage cytokines after the intratracheal instillation of bleomycin in rats. Am. Rev. Respir. Dis. 137,1135, 1988.

7. Kaelin, R.M., Center, D.M., Bernardo, J., Grant, M., and Snider, G.L. The role of macrophage-derived chemoattractants in the early inflammatory events of bleomycin-induced pulmonary fibrosis. Am. Rev. Respir. Dis. 128,132, 1983.

8. Kovacs, E.J., and Kelley, J. Secretion of macrophage-derived growth factor during acute lung injury induced by bleomycin. J. Leukocyte Biol. 37,1, 1985.

9. Lyman, S., Ujjani, B., Renner, K., Antholine, W., Petering, D.H., Whetsone, J.W., and Knight, J.M. Properties of the initial reaction of bleomycin and several of its metal complexes with Ehrlich cells. Cancer Res. 46,4472, 1986.

10. Munson, P.J., and Rodbard, D. LIGAND: A versatile computerized approach for characterization of ligand-binding systems. Anal. Biochem. 107,220, 1980.
11. Phan, S.H. Diffuse interstitial fibrosis. In Lung Biology in Health and Disease, Vol. 41: Lung Cell Biology, (D. Massaro, ed.) New York; Marcel Dekker, Inc., p. 907, 1989.

12. Pramila, S., and Hittelman, W.N. Kinetics and extent of repair of bleomycin-induced chromosome damage in quiescent normal human fibroblasts and human mononuclear blood cells. Cancer Res. 44,591, 1984.

13. Roy, S.N., and Band Horwitz, S. Characterization of the association of radiolabeled bleomycin $\mathrm{A}_{2}$ with $\mathrm{HeLa}$ cells. Cancer Res. 44,1541, 1984.

14. Schiffmann, E., Corcoran, B., and Wahl, S.M. N-Formylmethionyl peptides as chemoattractants for leucocytes. Proc. Natl. Acad. Sci. USA 72,1059, 1975.

15. Suwabe, A., Takahashi, K., Yasui, S., Arai, S., and Sendo, F. Bleomycin-stimulated hamster alveolar macrophages release interleukin-1. Am. J. Pathol. 132,512, 1988.

16. Tomoda, H., Kishimoto, Y., and Lee, Y.C. Temperature effect on endocytosis and exocytosis by rabbit alveolar macrophages. J. Biol. Chem. 264,15445, 1989.

17. Umezawa, H. Chemistry and mechanism of action of bleomycin. Fed. Proc. 33,2296, 1974.

18. Wright, S.D., and Jong, M.T.C. Adhesion-promoting receptors on human macrophages recognize Escherichia coli by binding to lipopolysaccharide. J. Exp. Med. 164,1876, 1986. 